\title{
Patient feedback and early outcome data with a novel tiered-binned model for multiplex breast cancer susceptibility testing
}

\author{
Angela R. Bradbury, MD ${ }^{1,2,3}$, Linda J. Patrick-Miller, PhD ${ }^{4,5}$, Brian L. Egleston, PhD ${ }^{6}$, \\ Laura DiGiovanni, MLA ${ }^{3}$, Jamie Brower, BS ${ }^{3}$, Diana Harris, MBe, PhD ${ }^{1}$, Evelyn M. Stevens, $\mathrm{MPH}^{1}$, \\ Kara N. Maxwell, MD, PhD' ${ }^{1}$ Abha Kulkarni'3 , Tyler Chavez ${ }^{1}$, Amanda Brandt, MS, \\ $\mathrm{CGC}^{1}$, Jessica M. Long, MS, CGC' ${ }^{1}$, Jacquelyn Powers, MS, CGC ${ }^{1}$, Jill E. Stopfer, MS, CGC ${ }^{3}$, \\ Katherine L. Nathanson, MD ${ }^{3,7,8}$ and Susan M. Domchek, MD ${ }^{1,3,8}$
}

\begin{abstract}
Purpose: The risks, benefits, and utilities of multiplex panels for breast cancer susceptibility are unknown, and new counseling and informed consent models are needed. We sought to obtain patient feedback and early outcome data with a novel tiered-binned model for multiplex testing.

Methods: $B R C A 1 / 2$-negative and untested patients completed preand posttest counseling and surveys evaluating testing experiences and cognitive and affective responses to multiplex testing.

Results: Of 73 patients, 49 (67\%) completed pretest counseling. $B R C A 1 / 2$-negative patients were more likely to proceed with multiplex testing $(86 \%)$ than those untested for BRCA1/2 $(43 \% ; P<$ $0.01)$. Many patients declining testing reported concern for uncertainty and distress. Most patients would not change anything about their pre- $(76 \%)$ or posttest $(89 \%)$ counseling sessions. Thirtythree patients (72\%) were classified as making an informed choice,
\end{abstract}

including $81 \%$ of those who proceeded with multiplex testing. Knowledge increased significantly. Anxiety, depression, uncertainty, and cancer worry did not significantly increase with multiplex testing.

Conclusion: Some patients, particularly those without prior $B R C A 1 / 2$ testing, decline multiplex testing. Most patients who proceeded with testing did not experience negative psychological responses, but larger studies are needed. The tiered-binned approach is an innovative genetic counseling and informed consent model for further study in the era of multiplex testing.

Genet Med advance online publication 2 April 2015

Key Words: Genetic counseling; genetic testing for cancer susceptibility; informed consent for genetic testing; multiplex genetic testing; tiered-binned genetic counseling

\section{INTRODUCTION}

Multiplex (i.e., multigene) panels including both high- and moderate-penetrance cancer susceptibility genes are currently being used in clinical practice despite questions regarding their clinical utility and no standard approach to genetic counseling and delivery. ${ }^{1-6}$ The inclusion of genes with varying penetrance and clinical utility has raised concerns. ${ }^{7}$ There are both advantages and disadvantages of multigene testing (as opposed to traditional phenotype-driven sequential testing), presenting challenges to patient education and informed decision making. ${ }^{3,7-10}$ The advantages and disadvantages of testing options must be shared with patients so they can make an informed decision regarding genetic testing. ${ }^{8,11-14}$ Traditional comprehensive models for pretest counseling and informed consent could be associated with information overload and poor informed decision making. ${ }^{7,15}$ In addition, multiplex testing has the potential to increase anxiety, uncertainty, and the adoption of inappropriate screening procedures or risk-reducing surgeries., ${ }^{3,7}$ Effective genetic education and counseling could minimize these risks and enhance adaptive responses to receiving multiplex results. ${ }^{13}$

This study has several aims. First, we sought to obtain patient feedback regarding the tiered-binned model for informed consent and counseling for multiplex testing among patients with a personal or family history of breast cancer. Second, we sought to begin to evaluate patient uptake of testing after pretest counseling and to evaluate informed decision making with the tiered-binned model. Third, we sought to begin to explore short-term cognitive and affective outcomes in clinical populations to better understand the potential risks, benefits, and utilities of incorporating multiplex genetic testing for breast cancer susceptibility assessment in clinical care.

\footnotetext{
${ }^{1}$ Division of Hematology-Oncology, Department of Medicine, University of Pennsylvania, Philadelphia, Pennsylvania, USA; ${ }^{2}$ Department of Medical Ethics and Health Policy, University of Pennsylvania, Philadelphia, Pennsylvania, USA; ${ }^{3}$ Abramson Cancer Center, University of Pennsylvania, Philadelphia, Pennsylvania, USA; ${ }^{4}$ ivision of HematologyOncology, Department of Medicine, The University of Chicago, Chicago, Illinois, USA; ${ }^{5}$ Center for Clinical Cancer Genetics and Global Health, The University of Chicago, Chicago, Illinois, USA; ${ }^{6}$ Fox Chase Cancer Center, Temple University Health System, Biostatistics and Bioinformatics Facility, Philadelphia, Pennsylvania, USA; ${ }^{7}$ Division of Translational Medicine and Human Genetics, Department of Medicine, University of Pennsylvania, Philadelphia, Pennsylvania, USA; ${ }^{8}$ Basser Research Center, Abramson Cancer Center, University of Pennsylvania, Philadelphia, Pennsylvania, USA. Correspondence: Angela R. Bradbury (angela.bradbury@uphs.upenn.edu)
} 


\section{MATERIALS AND METHODS}

\section{Participants}

Institutional review board approval was obtained and participants were recruited between December 2013 and March 2014. Two populations reflecting current clinical multiplex testing use were recruited: (i) patients who previously tested negative for $B R C A 1 / 2$ and (ii) patients presenting for BRCA1/2 testing. Both populations were candidates for myRisk, a 25-gene, nextgeneration sequencing-based test performed by Myriad Genetic Laboratories. Genes included are APC, ATM, BARD1, BMPR1A, BRCA1, BRCA2, BRIP1, CDH1, CDK4, CDKN2A, CHEK2, EPCAM (large rearrangements only), $M L H 1, M S H 2, M S H 6$, MUTYH, NBN, PALB2, PMS2, PTEN, RAD51C, RAD51D, SMAD4, STK11, and TP53. Patients new to BRCA1/2 testing had the cost of BRCA1/2 testing billed to insurance. Testing for other panel genes was covered by Myriad Genetic Laboratories.

Eligible participants were $\geq 18$ years old and could communicate in English. Similar to our prior studies of delivery innovations in cancer genetics, we recruited until we enrolled at least two individuals who had received a positive, variant of uncertain significance, and negative result. ${ }^{16,17}$ The sample size was calculated to obtain preliminary estimates with sufficient precision to power a subsequent study. We designed our study so that with $\sim 50$ participants our $95 \%$ confidence intervals would have precisions (i.e., half the width of the confidence interval) of $0.28 \mathrm{SD}$ units for continuous measures and $\leq 14 \%$ for binary measures. Potential participants were identified by clinical providers who shared an initial description of the study. Research staff introduced the study either in person or by phone and obtained written informed consent.

\section{Tiered-binned genetic counseling protocol and multiplex testing}

As previously described, we developed a tiered-binned genetic counseling approach for informed consent and genetic counseling for multiplex testing. ${ }^{7,18}$ In this model, tier 1 "indispensable" information is presented to all patients. More specific tier 2 information is provided to support variable informational needs among diverse patient populations. ${ }^{18}$ "Binning" clinically relevant information into groups is used to minimize information overload, support informed decision making, and facilitate adaptive responses to testing. Seven essential elements of informed consent are provided to address the unique limitations, risks, and uncertainties of multiplex testing. This model highlights various testing options for individual patients, including limited or targeted testing (e.g., BRCA1/2 only) or deferring testing for moderate-penetrance genes. ${ }^{18}$ For example, after pretest counseling participants could elect to decline the myRisk panel and consider other targeted genetic testing (e.g., BRCA1/2 only, TP53, PTEN, or Lynch syndrome genes, depending on personal and family history). Thirty-six standardized pretest and 15-22 (depending on test result and context) standardized posttest topics were developed to minimize differences among genetic counseling sessions. ${ }^{18}$ Genetic counselors underwent protocol training, including mock counseling sessions with individualized feedback. Genetic counselors completed counseling checklists, and sessions were audiotaped.

\section{Measures}

We used a novel conceptual mode ${ }^{17}$ grounded in the selfregulation theory of health behavior. ${ }^{19}$ Our model proposes that response to (i.e., psychosocial adjustment, uncertainty, perceived utility, informed decision making, satisfaction) and use of (performance of risk reduction and psychosocial behaviors) genomic information are products of an individual's understanding (i.e., knowledge and perception of the disease threat, the genetic test, and risk-reductive behaviors). ${ }^{17,20}$ Patients completed surveys assessing the theoretically informed constructs at baseline (T0), after pretest counseling (T1), and after receipt of results (T2).

Knowledge of genetic disease. Knowledge of genetic disease was evaluated (T0 and T1) using an adapted version combining the Cancer Genetics knowledge scale and ClinSeq knowledge scale. ${ }^{21-23}$ This scale includes three subscales evaluating knowledge of inheritance and test interpretation (eight items) and benefits (four items) and limitations (seven items) of multiplex testing (Cronbach $\alpha=0.80$ ). This scale also was used in the multidimensional measure of informed choice (MMIC), described further below.

Psychosocial adjustment. Psychosocial adjustment was evaluated (T0 through T2) with three measures. (i) State anxiety was measured with the 20 -item State Inventory of the StateTrait Anxiety Inventory ${ }^{24,25}$ (Cronbach $\alpha=0.92$ ). (ii) General anxiety and depression were assessed with the seven-item Hospital Anxiety and Depression Scale anxiety and depression subscales $^{26}$ (Cronbach $\alpha=0.87$ and 0.84 , respectively). (iii) Breast cancer worry was evaluated with 14 items of the Impact of Events Scale (Cronbach $a=0.90) .{ }^{27}$ We excluded one item lacking face validity in our population.

Satisfaction with genetic services. Satisfaction with genetic services was measured (T1 and T2) with an established 13item scale evaluating participants' perceptions of their genetic counseling and testing experience, including cognitive, affective, and time/attention items ${ }^{16,28}$ (Cronbach $\left.\alpha=0.85\right)$.

Uncertainty. Uncertainty was assessed (T0 through T2) using a three-item scale adapted from the Multidimensional Impact of Cancer Risk Assessment questionnaire ${ }^{29}$ (Cronbach $\alpha=0.88$ ).

Perceived utility. Perceived utility was assessed (T1 and T2) with a novel scale developed to evaluate patient perceptions of the utility of genomic information for their life and the lives of their family members. It comprises 12 items evaluating medical and personal utility on a five-point Likert scale (Cronbach $\alpha=0.90$ ).

Attitudes about genetic testing. Attitudes about genetic testing were assessed at T1 with an eight-item scale evaluating overall attitudes about testing after pretest counseling and used as described below in the MMIC (Cronbach $\alpha=0.73$ ). ${ }^{30,31}$

Opinions and experiences regarding genetic counseling and delivery. Opinions and experiences regarding genetic counseling and delivery were assessed (T1 and T2) with openended questions to elicit opinions regarding and suggestions for 
improving the counseling model. Participants also were asked to indicate (on a five-point Likert scale) their willingness to receive their counseling sessions through other methods (phone and videoconference).

\section{Statistical analyses}

Framework analysis was used to analyze open-ended responses. ${ }^{32}$ Two research staff (D.H., E.M.S.) independently reviewed responses, using thematic analysis to record primary and secondary themes for each item. Disagreements in coding assignments were resolved by a third reviewer (A.R.B.).

Informed decision making was defined using the MMIC, published and validated by Michie et al. ${ }^{31}$ and Marteau et al. ${ }^{33}$ While decisional conflict is often used to evaluate decision making with genetic testing, we elected to use the MMIC to assess informed decision making. The MMIC incorporates three important dimensions of choice, including knowledge as well as individual values and attitudes toward choice options and the extent that choice reflects attitude. Informed choice is grounded in the principles of autonomy and respect for persons and requires that a choice be voluntary and that an individual understand the potential outcomes of the decision and alternative choices. We used the MMIC because it incorporates these important theoretical constructs of informed choice. Consistent with prior applications of the MMIC, adequate knowledge of genetic diseases is defined as correct answers to $\geq 50 \%$ of the items, and a positive attitude about genetic testing was indicated by a score at or above the midpoint of the scale. ${ }^{30,31}$ A more informed choice is defined by adequate knowledge and a testing decision concordant with attitudes about genetic testing (e.g., adequate knowledge/positive attitude toward testing/decision to proceed with testing or adequate knowledge/negative attitude toward testing/decision to decline testing). All other categories are considered less informed.

Predefined criteria were developed to select audiotaped sessions for review to inform modifications to the tiered-binned counseling protocol. ${ }^{16}$ These included (i) all sessions disclosing a positive result $(n=5)$; (ii) provider request because of several session interruptions ( $n=1$ pretest session); (iii) qualitative responses suggesting communication difficulties or less optimal outcomes ( $n=13$ pretest sessions, $n=2$ posttest sessions); (iv) decline in knowledge or satisfaction in the lowest 10th percentile, ( $n=10$ pretest sessions, $n=8$ posttest sessions); and (v) increase in state anxiety, cancer worry, general anxiety, or depression in the highest 10 th percentile ( $n=14$ pretest sessions, $n=7$ posttest sessions). Sessions for all patients who were classified as making less informed decisions were reviewed ( $n=13$ pretest sessions). These criteria resulted in review of 32 (65\%) and 17 (52\%) pretest and posttest sessions, respectively.

We calculated means, SDs, and proportions for all constructs and evaluated longitudinal changes from baseline to after disclosure (T0 to T2). We used Fisher exact tests and Wilcoxon tests for pairwise comparisons. For longitudinal analyses we used multiple linear regressions estimated by generalized estimating equations with robust SEs. We assumed exchangeable correlation matrices for the models estimated with generalized estimating equation. $P$ values $<0.05$ based on two-sided hypothesis tests were considered statistically significant.

\section{RESULTS}

\section{Participant characteristics}

Of the 73 eligible subjects approached, 49 (67\%) consented to participate in the study (Figure 1). Among those who declined, $30 \%$ did so because they were concerned about the potential for uncertainty or unclear utility. Decliner rates did not differ between BRCA1/2-negative and BRCA1/2-untested participants. Participant characteristics are described in Table 1. Those who enrolled were more likely to have a history of cancer. $B R C A 1 / 2$-untested patients were more likely to be nonwhite, have no personal history of cancer, and have lower knowledge of testing benefits. All counseling sessions were in person (average 49 and 20 minutes for pretest and posttest sessions, respectively). Patients also met with a physician during posttest sessions to discuss medical recommendations. Postvisit survey completion rates were high (98\% at T1 and 95\% at T2). Genetic counselor fidelity to communication topics was high (pretest: mean 73\%; posttest: mean $73-100 \%$, depending on the result). The most frequently omitted items included emotional assessments and the teach-back, ${ }^{34,35}$ in which patients describe what they understand from the session.

\section{Uptake of multiplex testing}

Forty-nine participants (67\%) enrolled and completed pretest counseling (Figure 1). After pretest counseling, BRCA1/2negative patients were more likely to proceed with multiplex testing than those untested for $B R C A 1 / 2$ ( 86 vs. $43 \% ; P<0.01$ ). Among the $11 B R C A 1 / 2$-untested patients who declined multiplex testing, $82 \%$ proceeded with $B R C A 1 / 2$ testing alone. One patient elected to proceed with testing for $B R C A 1 / 2$ and a panel for Lynch syndrome. The most frequent reasons for declining multiplex testing were that BRCA1/2 testing was most relevant to medical decision making and that testing beyond BRCA1/2 was overwhelming and not something the patient had mentally prepared for (in particular testing for TP53). BRCA1/2-negative participants reported declining multiplex testing $(n=3)$ because of a lack of clear clinical utility and the potential for distress with uncertainty.

\section{Informed decision making}

Of the participants, $72 \%(n=33)$ were defined as making an informed choice (adequate knowledge and a decision concordant with attitudes toward genetic testing) about genetic testing options (Table 2). This did not differ significantly between groups (77\% among BRCA1/2-negative participants; $65 \%$ among those untested for $B R C A 1 / 2)$. Among those who specifically proceeded with multiplex testing, 25 (81\%) were defined as making an informed choice. Making a less informed choice was associated with greater breast cancer worry $(P=0.03)$, greater uncertainty $(P=0.06)$, and greater perceived utility $(P$ $<0.01)$ after pretest counseling. Making a less informed choice was associated with lower counselor-reported use of emotional 


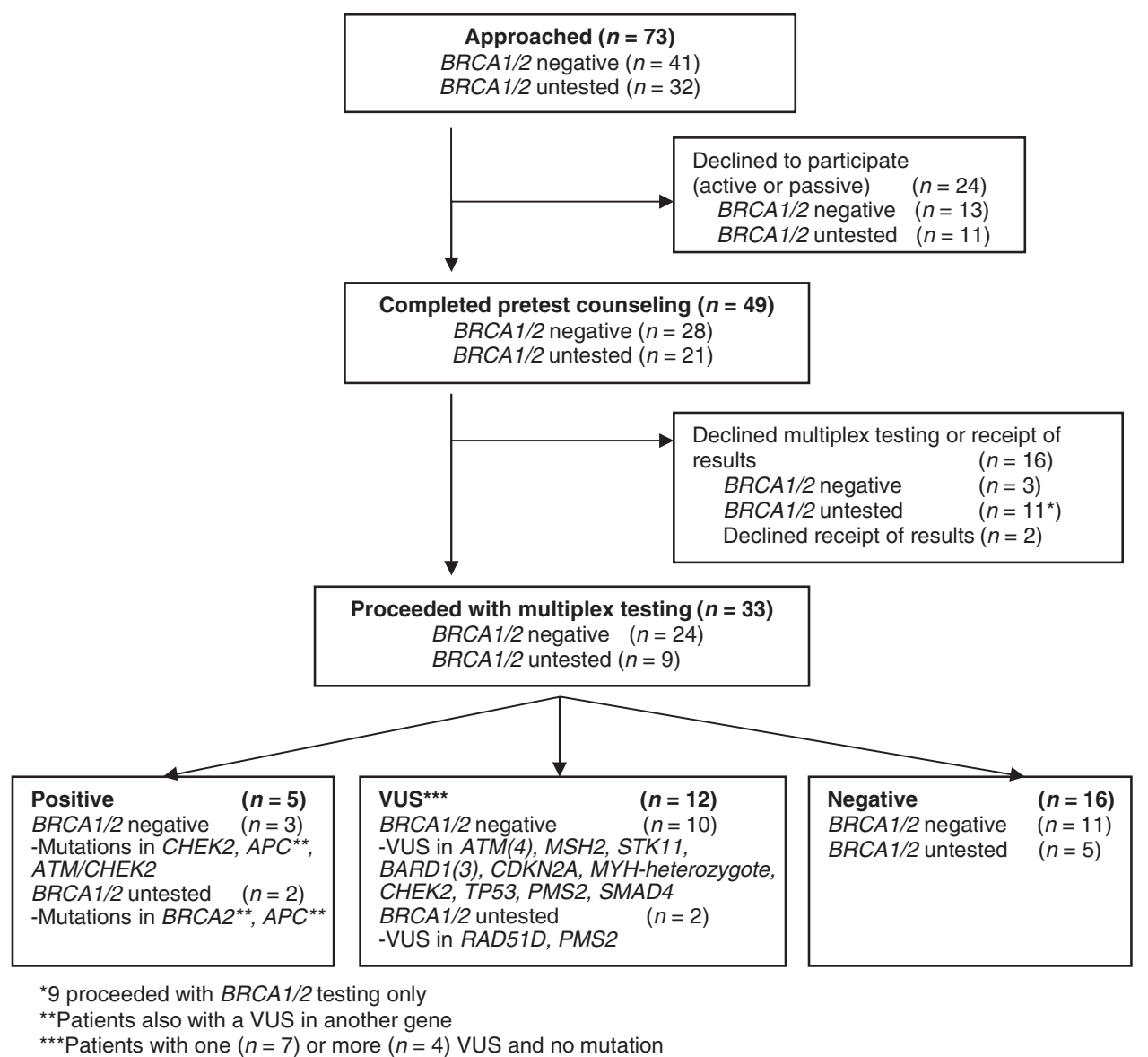

Figure 1 Study flow chart.

and knowledge assessments, although these differences were not statistically significant.

\section{Patient feedback}

Most patients reported they would not change anything about their pretest $(76 \%)$ or posttest (89\%) session (Table 3 ). Patients indicated that they found sessions to be informative and that they valued the engagement of the tiered approach, as well as the clarity and simplicity of the information presented. Potential refinements to sessions based on participant responses included enhancing assessments of informational overload, confusion, and psychosocial needs, in particular when associated with uncertain results.

Only $30 \%$ of participants indicated they would have been willing to receive their pretest session by phone. Many described the benefits of face-to-face communication for complex information, psychosocial support, and visual aids, which would be harder to use by phone. More (66\%) reported they would have been willing to receive pretest session by videoconference in their home. After posttest counseling, 41\% and $43 \%$ indicated that they would have been willing to receive their results by phone or videoconference, respectively.

\section{Audiotape review}

Forty-nine counseling sessions (65\% of pretest sessions; $52 \%$ of posttest sessions) were reviewed by a clinical health psychologist
(L.P.M.) who, assimilating them with the patient feedback, identified several potential modifications to enhance outcomes of the tiered-binned counseling protocol. These included training providers to increase awareness of biopsychosocial risk factors for less optimal outcomes (e.g., baseline general and cancer-specific anxiety and familial experiences) and the use of affective probes throughout sessions. Other modifications that could enhance understanding of complex information and reduce uncertainty include greater inclusion of visual aids, the use of lay language and the teach-back technique, and addressing patient concerns about privacy of genetic information and potential costs.

\section{Outcomes of multiplex testing}

As shown in Table 4, event anxiety, depression, cancer worry, and uncertainty did not significantly change among all participants or the subset that proceeded with multiplex testing. General anxiety decreased significantly after pretest counseling, and total knowledge and knowledge of the limitations of testing increased significantly across all time points (Table 4). Satisfaction was modest and did not change significantly after the receipt of test results. There were no significant differences in these outcomes by test result (e.g., positive versus variant of uncertain significance versus negative) or by exposure to testing (e.g., BRCA1/2 negative versus untested). Exploratory evaluation of counselor-reported use of the teach-back (inclusion/ omission) with cognitive and affective outcomes suggest the 
Table 1 Participant characteristics

\begin{tabular}{|c|c|c|c|c|}
\hline \multirow[b]{2}{*}{ Characteristics } & \multicolumn{4}{|c|}{ Participants } \\
\hline & $\begin{array}{l}\text { Approached } \\
(n=73)(\%)\end{array}$ & $\begin{array}{c}\text { Enrolled } \\
(n=49)(\%)\end{array}$ & $\begin{array}{c}\text { BRCA1/2 negative } \\
(n=28)(\%)\end{array}$ & $\begin{array}{c}\text { BRCA1/2 untested } \\
(n=21)(\%)\end{array}$ \\
\hline \multicolumn{5}{|l|}{ Age, years } \\
\hline Median & 50 & 50 & 50 & 53 \\
\hline Range & $29-80$ & $36-71$ & $38-71$ & $36-68$ \\
\hline Black & $6(8)$ & $4(8)$ & $0(0)$ & $4(19)$ \\
\hline Hispanic/Latino, mixed & $4(6)$ & $3(6)$ & $3(11)$ & $0(0)$ \\
\hline \multicolumn{5}{|l|}{ Gender } \\
\hline Female & $71(97)$ & $47(96)$ & $27(96)$ & $20(95)$ \\
\hline Male & $2(3)$ & $2(4)$ & $1(4)$ & $1(5)$ \\
\hline College graduate & $14(27)$ & $14(30)$ & $6(21)$ & $8(38)$ \\
\hline Graduate or postgraduate & $27(53)$ & $23(49)$ & $14(50)$ & $9(43)$ \\
\hline Missing data & $22(-)$ & $2(-)$ & $1(-)$ & $1(-)$ \\
\hline \multicolumn{5}{|l|}{ Married ${ }^{a}$} \\
\hline Yes & $45(77)$ & $36(77)$ & $21(75)$ & $15(71)$ \\
\hline Personal history of cancer & $58(79)$ & $38(78)$ & $25(89)$ & $13(62)$ \\
\hline Breast cancer only & $48(66)^{c}$ & $35(71)^{c}$ & $23(82)$ & $12(52)$ \\
\hline Other cancer ${ }^{d}$ & $3(4)^{c}$ & $0(0)^{c}$ & $0(0)$ & $0(0)$ \\
\hline Multiple primaries & $7(10)^{c}$ & $3(6)^{c}$ & $2(7)$ & $1(5)$ \\
\hline First- or second-degree relative with cancer (mean, SD) & $3.4(2.3)$ & $3.7(2.4)$ & $3.3(2.4)$ & $4.1(2.5)$ \\
\hline Knowledge limitations (range 6-30) & - & $20.4(3.2)$ & $20.6(3.5)$ & $20.1(2.7)$ \\
\hline General depression (range 0-21) & - & $2.6(2.9)$ & $2.4(2.9)$ & $3(3.1)$ \\
\hline General anxiety (range 0-21) & - & $6.9(3.9)$ & $6.6(3.9)$ & $7.2(3.9)$ \\
\hline State anxiety (range 20-80) & - & $37.6(10.0)$ & $37.4(10.9)$ & $37.9(9.1)$ \\
\hline Breast cancer worry (range 0-75) & - & $18.0(15.4)$ & $16.0(15.4)$ & $20.7(15.3)$ \\
\hline Uncertainty (range 0-15) & - & $7.5(4.2)$ & $7.1(4.5)$ & $8(3.8)$ \\
\hline
\end{tabular}

Data are $n(\%)$ unless otherwise indicated.

${ }^{a}$ Twenty-two participants with missing data among approached; 2 enrolled with missing data. ${ }^{\mathrm{b}} P<0.01 .{ }^{c} p=0.03$. ${ }^{\mathrm{d}}$ Ovarian $(n=2)$, colon $(n=1)$. eMultiple primary cancers included breast $(n=6)$, ovarian $(n=1)$, esophageal $(n=1)$, sarcoma $(n=1)$, lung $(n=1)$, endometrial $(n=1)$, and thyroid $(n=3)$. ${ }^{f} P<0.02$.

potential for better outcomes (less anxiety, better knowledge) after disclosure when the teach-back is not omitted during disclosure sessions.

Only $15 \%(n=5)$ of patients who proceeded with testing had a positive result (Figure 1 ). This included only one mutation in a high-penetrance gene (BRCA2). Medical management changed based on the genetic test result in only two patients (5\%) (Supplementary Table S1 online). Testing for relatives was recommended only for patients with mutations in high-penetrance genes (e.g., BRCA2). Given uncertainty regarding the remaining cancer risk in individuals who test negative for a familial mutation in moderate-penetrance genes, we offered relatives in these cases the opportunity to discuss the limitations of testing for the familial mutation and how it may or may not change medical management for them. Patientreported perceived utility was modest after pretest counseling, and mean scores declined after receipt of results, although this was not statistically significant. Sixteen patients (42\%) reported 
Table 2 Informed decision making with the tiered-binned model

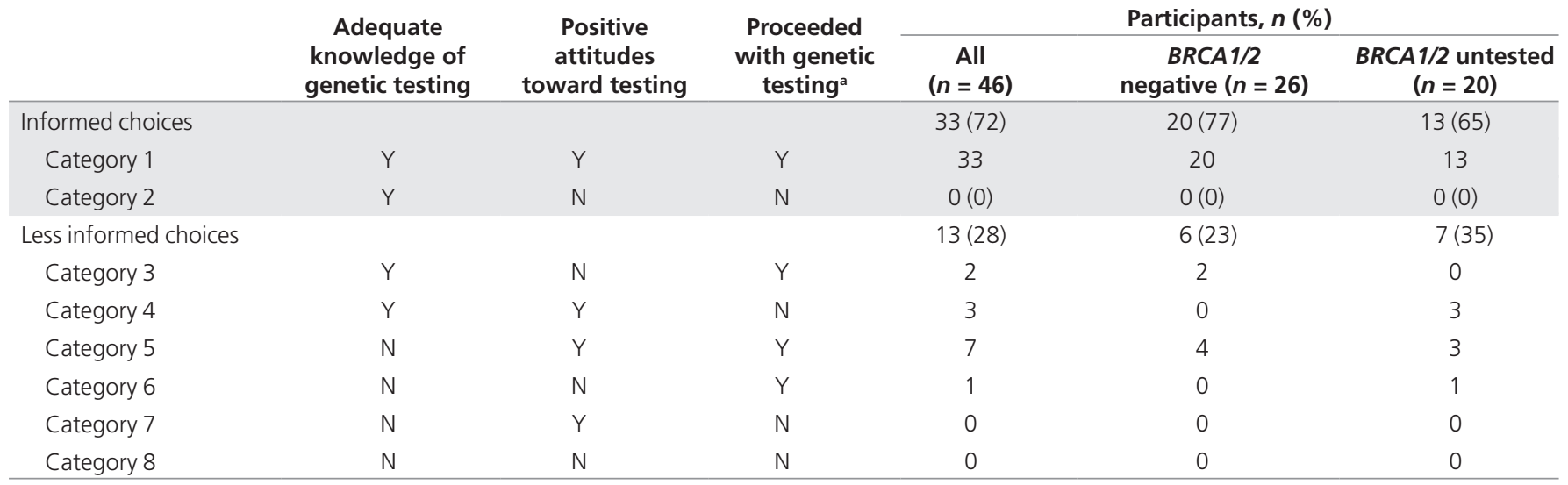

Two patients had missing baseline data and were excluded from analyses.

$\mathrm{N}, \mathrm{no}$; $\mathrm{Y}$, yes.

ancluding $B R C A 1 / 2$ only or multiplex testing for those without prior testing.

Table 3 Patient open-ended responses regarding the tiered-binned model for genetic counseling and informed consent

\section{Coded themes ${ }^{\text {a }}$}

What did you like about your counseling session?

Informative for self or relatives (e.g., "talked about what they could possibly find from the testing, it was all spelled out really well in what to expect")

Engaging (e.g., "teach-back technique-she asked me to give her a summary of what information she shared with me. Reinforced the knowledge I gained" and "it was more of a conversation where I could ask questions and interject along the way")

Comprehensible (e.g., "the information was presented in a very straightforward and sequential way. I really liked the visual aids; they helped me to learn what I needed to learn")

Comprehensive (e.g., "summarized the implications of the results, confirmed what I had thought I had learned prior to the testing, and I was able to ask some extra questions")

Straightforward (e.g., "I liked that it was concise and to the point")

What did you dislike about your counseling session?

Nothing

Uncertainty (e.g., "the last question I think they asked was what the results mean to me. I had to be blunt because I had to say they were meaningless")

Overwhelming (e.g., "A lot of information to absorb. Kinda need a genetics 101 refresher")

Distressing (e.g., "There's a black cloud over my head if I don't get the testing because it's like you're constantly wondering what is causing it, but it's a little black cloud if I get it the testing and they find something because then I know it's genetic and there's nothing I can do")

Missing information (e.g., "Unknowns regarding payments, fees, coverage. . ")

What would you change about your counseling session?

Nothing

Provide more information regarding new research, medical management, basic genetics (e.g., "Add some ready-prework while waiting to get into the appointment with understanding the basics on genetics, keeping it at a low literacy level")

Data are $n(\%)$

aRestricted to themes specific to the counseling model. We excluded themes about providers (personable, patient, knowledgeable), the setting, and process or research procedures (space, wait, surveys).

a significant increase in perceived utility after testing, including three of the five with positive results. Increases in perceived utility were highest among those receiving a positive result (positive $=60 \%$; variant of uncertain significance $=20 \%$, negative $=48 \%)$, although these differences were not statistically significant.
After pretest counseling $(n=46)$

After posttest counseling $(n=39)$

$\begin{array}{ll}17(37) & 11(28) \\ 17(37) & 5(13) \\ 14(30) & 4(10) \\ 6(13) & 2(5) \\ 2(4) & 4(10)\end{array}$

$\begin{array}{cr}28(60) & 24(62) \\ 0(0) & 8(21) \\ 4(9) & 0(0) \\ 3(7) & 3(7)\end{array}$

$3(7)$

$38(76)$

35 (89)

2 (4)

2 (5) 
Table 4 Short-term cognitive and affective outcomes

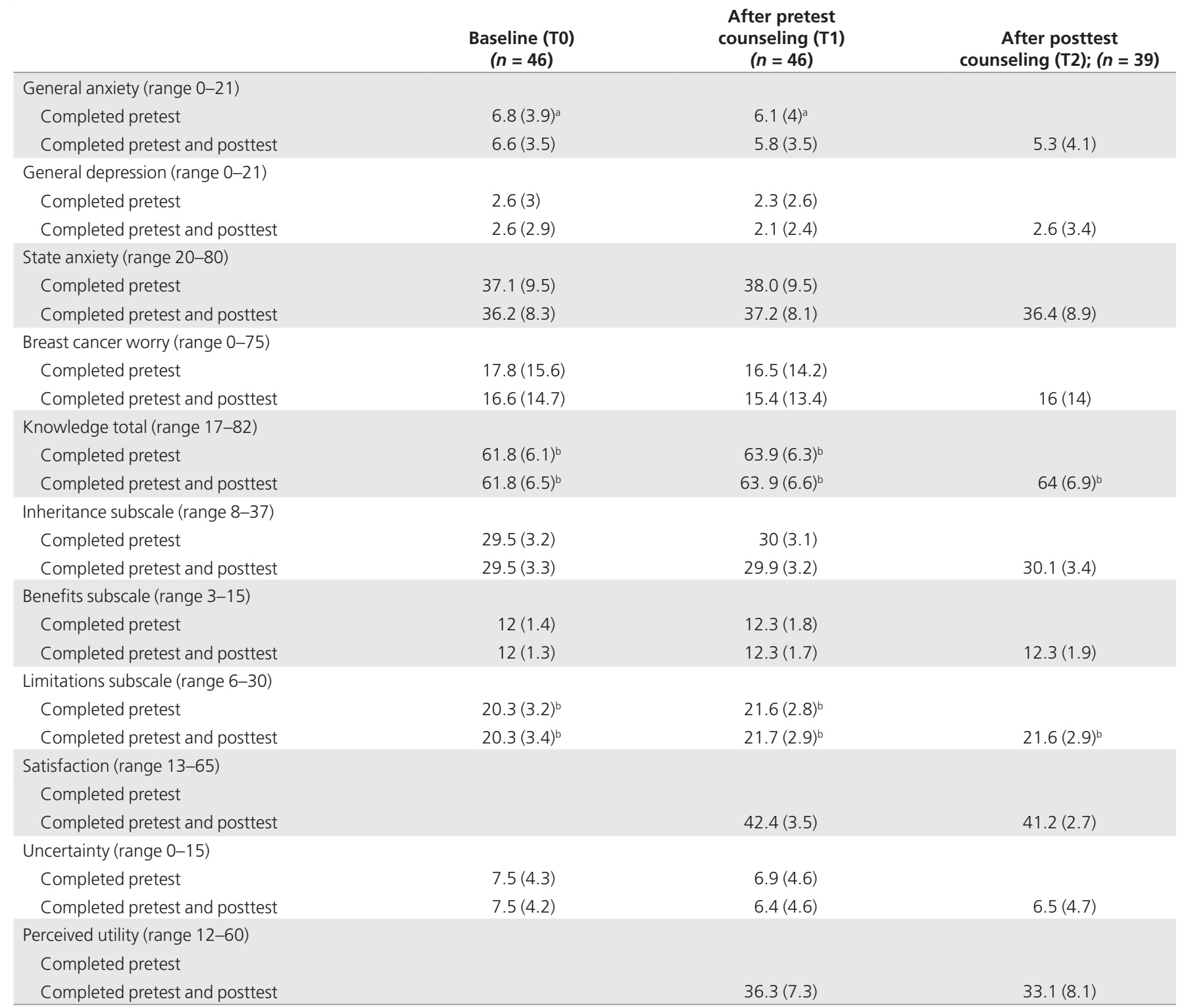

Data are mean (SD).

a $P<0.05$ for all participants only. Not statistically significant for the subset who proceeded with multiplex testing. ${ }^{b} P<0.001$ for all participants and the subset who proceeded with multiplex testing.

testing including genes of variable penetrance and utility. Patient feedback suggests that patients value the engagement and organizational approach to sharing complex information in the tiered-binned model. Although most patients would not change anything about their counseling experiences, some did provide suggestions for refinements, which align with the observations from the audiotape review of sessions selected for less optimal outcomes. Modifications to further enhance patient experiences include considering baseline patient risk factors for distress, ${ }^{36}$ adherence to use of verbal affective and knowledge probes and the teach-back strategy during disclosure sessions, inclusion of visual aids, and substitution of lay language for medical terms. In addition, while addressing privacy and discrimination risks are a standard component of genetic pretest counseling included in the tiered-binned model, ensuring that privacy issues are iterated throughout patient encounters might enhance counselor-patient communication and patient outcomes.

A key finding in this study is that many patients declined multiplex testing. Uptake was lowest among patients referred for $B R C A 1 / 2$ testing (i.e., not previously tested). While the majority of patients were defined as making informed decisions, those patients who had not undergone prior BRCA1/2 testing were less likely to meet criteria for an informed decision. Although the subgroups are small, the baseline cognitive and affective factors among BRCA1/2-negative and -untested groups did not differ significantly. Nonetheless, these two patient populations might differ in their susceptibility to 


\section{ORIGINAL RESEARCH ARTICLE}

information overload and tolerance for uncertainty. ${ }^{25}$ Our data suggest there might be value in sequential testing for some patients, supporting additional studies designed to understand potential differences by prior testing exposure and patient characteristics. Understanding testing decisions in the setting of multiple available testing options is an important area for further study. Defining optimal pretest counseling approaches to support informed decision making will be important for establishing clinical guidelines, particularly during the early phase of clinical incorporation of multiplex testing, when there is a higher likelihood of uncertainty and unclear clinical utility, and in the setting of multiple testing options.

In this initial study using a novel tiered-binned counseling model, the majority of patients were classified as making informed decisions, which was defined as having adequate knowledge and making testing decisions concordant with their attitudes toward genetic testing. This was also the case for those who specifically proceeded with multiplex testing. Further, knowledge-in particular knowledge of the limitations of multiplex testing-increased following the tiered-binned pretest counseling model. Understanding the limitations of genetic testing is a key component of informed consent ${ }^{14,18}$ and is particularly important in the setting of genetic tests with variable utility and uncertain risks. ${ }^{3,7,37}$ Thus, these patient-reported data suggest that this new tiered-binned approach to genetic counseling has the potential to increase knowledge and to support informed decision making in the context of next-generation sequencing, although further larger studies designed to evaluate outcomes and the clinical application of this model are warranted.

These initial results suggest that most patients who proceed with multiplex testing after the tiered-binned counseling approach do not experience significant short-term increases in uncertainty and distress, although this may vary by test result, counseling approach, the delivery model and over time. ${ }^{25}$ Larger, prospective, longitudinal studies are needed to confirm these results and to better understand how outcomes vary for individuals and by test result. While telephone has been increasingly utilized for delivery of $B R C A 1 / 2$ genetic counseling and testing and seems to have equivalent outcomes for those who proceed with testing, ${ }^{38,39}$ many patients expressed concern with the use of alternative delivery models in this setting. Thus, outcomes of telephone and videoconferencing counseling and disclosures in the setting of multiplex testing will need to be studied further. Videoconferencing has the additional benefit of face-to-face communication, providing potentially higher awareness of nonverbal cues, which could be an advantage over the telephone, particularly in the setting of complex medical information. Current potential disadvantages to real-time videoconferencing include technology challenges, privacy, and costs. In addition, our results reflect outcomes with the tieredbinned approach, and outcomes may differ depending on the content included in, the extent of, and the approach to pretest education and counseling. Comparing outcomes with varying counseling approaches could be useful to establish best practices and optimize outcomes.
Even with data suggesting limited negative affective responses, the utility of large-scale adoption of multiplex testing for breast cancer susceptibility remains uncertain. Although some patients have documented changes in medical management, ${ }^{4}$ how often multiplex testing results in a change beyond what would have been recommended based on personal and family history alone remains unclear and will require larger prospective studies. ${ }^{40}$ Even if test results do not change what medical providers recommend, a positive genetic test result might result in greater screening compliance or adoption of risk-reducing interventions. ${ }^{7}$ In addition, there may be benefits to family members, although these will vary by gene and by family history. Alternatively, uncertainty, misunderstanding, and anxiety might prompt inappropriate adoption of risk-reducing or screening interventions. In addition to clinical utility, there may be additional utilities (e.g., personal, future, economic) of interest in considering the benefits and risks of clinical incorporation of multiplex testing. ${ }^{\text {? }}$ Thus, studies evaluating both psychosocial outcomes and performance of health behaviors and various utilities of multiplex testing are needed.

We acknowledge several limitations of this study. This is a small study designed to inform modifications to a novel counseling model, and results will need to be confirmed in larger studies designed to evaluate testing outcomes. These studies are currently ongoing. ${ }^{18}$ We had few positive or unexpected results, which could be associated with more anxiety, uncertainty, or perceived utility. Larger prospective, longitudinal studies to evaluate differences by subgroups are needed and are ongoing. Our population is highly educated, and results may not be representative of more diverse clinical populations. While the majority of patients made informed decisions, the modifications suggested by audiotape reviews and patient feedback may or may not improve outcomes. In addition, alternative counseling models may be equally or more effective or more efficient given a limited genetic provider workforce and increased incorporation of genetics into clinical care. This counseling model was specifically developed for breast cancer susceptibility panels, which include both high- and moderate-penetrance genes, and may not be applicable to other cancer syndromes.

In conclusion, some patients decline multiplex testing, in particular those without prior $B R C A 1 / 2$ testing, highlighting the importance of informed consent. Early data suggest the tiered-binned genetic counseling model is associated with increased knowledge and informed decisions in the majority of patients, representing a potential strategy for further study in the era of multiplex testing. Although most patients did not experience negative psychological responses, larger studies are needed to evaluate subgroup differences and to better understand the risks, benefits, and utilities of multigene testing for cancer susceptibility.

\section{SUPPLEMENTARY MATERIAL}

Supplementary material is linked to the online version of the paper at http://www.nature.com/gim 


\section{ACKNOWLEDGMENT}

This research was supported by the Rooney Family Fund. The cost of genetic testing was covered, in part, by Myriad Genetics. Myriad Genetics did not otherwise provide financial support for the study.

\section{DISCLOSURE}

A.R.B. and S.M.D. report that Myriad Genetics is covering the cost of multiplex testing in this study and an ongoing longitudinal study. The other authors declare no conflict of interest.

\section{REFERENCES}

1. Rainville IR, Rana HQ. Next-generation sequencing for inherited breast cancer risk: counseling through the complexity. Curr Oncol Rep 2014;16:371.

2. Lundy MG, Forman A, Valverde K, Kessler L. An investigation of genetic counselors' testing recommendations: pedigree analysis and the use of multiplex breast cancer panel testing. J Genet Couns 2014;23:618-632.

3. Domchek SM, Bradbury A, Garber JE, Offit K, Robson ME. Multiplex genetic testing for cancer susceptibility: out on the high wire without a net? J Clin Oncol 2013;31:1267-1270.

4. Kurian AW, Hare EE, Mills MA, et al. Clinical evaluation of a multiplegene sequencing panel for hereditary cancer risk assessment. J Clin Oncol 2014;32:2001-2009.

5. Mauer CB, Pirzadeh-Miller SM, Robinson LD, Euhus DM. The integration of next-generation sequencing panels in the clinical cancer genetics practice: an institutional experience. Genet Med 2014;16:407-412.

6. Burke W, Dimmock D. Clinical decisions. Screening an asymptomatic person for genetic risk. N Engl J Med 2014;370:2442-2445.

7. Bradbury AR, Patrick-Miller L, Domchek S. Multiplex genetic testing: reconsidering utility and informed consent in the era of next-generation sequencing. Genet Med 2014;17:97-98.

8. Sharp RR. Downsizing genomic medicine: approaching the ethical complexity of whole-genome sequencing by starting small. Genet Med 2011;13:191-194.

9. Wolfe Schneider K, Anguiano A, Axell L, et al. Collaboration of colorado cancer genetic counselors to integrate next generation sequencing panels into clinical practice. J Genet Couns 2014:23:640-646.

10. Fecteau H, Vogel KJ, Hanson K, Morrill-Cornelius S. The evolution of cancer risk assessment in the era of next generation sequencing. J Genet Couns 2014;23:633-639.

11. Robson ME, Storm CD, Weitzel J, Wollins DS, Offit K; American Society of Clinical Oncology. American Society of Clinical Oncology policy statement update: genetic and genomic testing for cancer susceptibility. J Clin Oncol 2010;28:893-901.

12. Trepanier AM, Allain DC. Models of service delivery for cancer genetic risk assessment and counseling. J Genet Couns 2014;23:239-253.

13. Biesecker BB. Goals of genetic counseling. Clin Genet 2001;60:323-330.

14. Elias S, Annas GJ. Generic consent for genetic screening. N Engl J Med 1994;330:1611-1613.

15. Berg JS, Khoury MJ, Evans JP. Deploying whole genome sequencing in clinical practice and public health: meeting the challenge one bin at a time. Genet Med 2011: 13:499-504.

16. Patrick-Miller L, Egleston BL, Daly M, et al. Implementation and outcomes of telephone disclosure of clinical BRCA1/2 test results. Patient Educ Couns 2013;93:413-419.

17. Patrick-Miller $L$, Egleston $B L$, Fetzer $D$, et al. Development of a communication protocol for telephone disclosure of genetic test results for cancer predisposition. JMIR Res Protoc 2014;3:e49.

18. Bradbury AR, Patrick-Miller L, Long J, et al. Development of a tiered and binned genetic counseling model for informed consent in the era of multiplex testing for cancer susceptibility. Genet Med; (e-pub ahead of print) 9 October 2014.
19. Leventhal $H$, Benyamini $Y$, Brownlee $S$, et al. Perceptions of health and illness: current research and applications. In: Petrie KJ, Weinman JA (eds). Illness Representations: Theoretical Foundations. Harwood: Amsterdam, The Netherlands, 1997: 19-46.

20. Shiloh S. Illness representations, self-regulation, and genetic counseling: a theoretical review. J Genet Couns 2006;15:325-337.

21. Kaphingst KA, McBride CM, Wade $C$, et al. Patients' understanding of and responses to multiplex genetic susceptibility test results. Genet Med 2012;14:681-687.

22. Lerman C, Narod S, Schulman K, et al. BRCA1 testing in families with hereditary breast-ovarian cancer. A prospective study of patient decision making and outcomes. JAMA 1996;275:1885-1892.

23. Kelly K, Leventhal H, Marvin M, Toppmeyer D, Baran J, Schwalb M. Cancer genetics knowledge and beliefs and receipt of results in Ashkenazi Jewish individuals receiving counseling for BRCA1/2 mutations. Cancer Control 2004;11:236-244.

24. Speilberger CD, Gorsuch RL, Lushene R, et al. Manual for the State-Trait Anxiety Inventory. Consulting Psychologists Press: Palo Alto, CA, 1983.

25. Hamilton JG, Lobel M, Moyer A. Emotional distress following genetic testing for hereditary breast and ovarian cancer: a meta-analytic review. Health Psychol 2009;28:510-518

26. Zigmond AS, Snaith RP. The hospital anxiety and depression scale. Acta Psychiatr Scand 1983;67:361-370.

27. Horowitz M, Wilner N, Alvarez W. Impact of Event Scale: a measure of subjective stress. Psychosom Med 1979;41:209-218.

28. Pieterse $A H$, van Dulmen AM, Beemer FA, Bensing JM, Ausems MG. Cancer genetic counseling: communication and counselees' post-visit satisfaction, cognitions, anxiety, and needs fulfillment. J Genet Couns 2007;16:85-96.

29. Cella D, Hughes C, Peterman A, et al. A brief assessment of concerns associated with genetic testing for cancer: the Multidimensional Impact of Cancer Risk Assessment (MICRA) questionnaire. Health Psychol 2002;21:564-572.

30. O'Neill SC, White DB, Sanderson SC, et al. The feasibility of online genetic testing for lung cancer susceptibility: uptake of a web-based protocol and decision outcomes. Genet Med 2008;10:121-130.

31. Michie S, Dormandy E, Marteau TM. The multi-dimensional measure of informed choice: a validation study. Patient Educ Couns 2002;48:87-91.

32. Ritchie J, Spencer L. Qualitative data analysis for applied policy research. In: Bryman A, Burgess R (eds). Analysing Qualitative Data. Routledge: London, 1994: 173-194.

33. Marteau TM, Dormandy E, Michie S. A measure of informed choice. Health Expect 2001;4:99-108.

34. Ubel PA. Critical Decisions: How You and Your Doctor can Make the Right Medical Choices Together. HarperCollins: New York, 2012.

35. Geller G, Botkin JR, Green MJ, et al. Genetic testing for susceptibility to adult-onset cancer. The process and content of informed consent. JAMA 1997;277:1467-1474.

36. Eijzenga W, Aaronson NK, Hahn DE, et al. Effect of routine assessment of specific psychosocial problems on personalized communication, counselors' awareness, and distress levels in cancer genetic counseling practice: a randomized controlled trial. J Clin Onco/ 2014;32:2998-3004.

37. Bunnik EM, de Jong A, Nijsingh N, de Wert GM. The new genetics and informed consent: differentiating choice to preserve autonomy. Bioethics 2013;27: 348-355.

38. Kinney AY, Boonyasiriwat W, Walters ST, et al. Telehealth personalized cancer risk communication to motivate colonoscopy in relatives of patients with colorectal cancer: the family CARE Randomized controlled trial. J Clin Oncol 2014;32:654-662.

39. Schwartz MD, Valdimarsdottir HB, Peshkin BN, et al. Randomized noninferiority trial of telephone versus in-person genetic counseling for hereditary breast and ovarian cancer. J Clin Oncol 2014;32:618-626.

40. Domchek SM, Nathanson KL. Panel testing for inherited susceptibility to breast, ovarian, and colorectal cancer. Genet Med 2014;16:827-829. 